\title{
Evaluación del desempeño de un amortiguador de masa sintonizado no lineal mediante simulaciones híbridas en tiempo real
}

\section{Performance evaluation of a non-linear tuned mass damper through real-time hybrid simulation}

DOI: 10.17981/ingecuc.15.2.2019.02

Artículo de Investigación Científica. Fecha de Recepción:04/09/2018, Fecha de Aceptación:20/05/2019.

\author{
Carlos Andrés Riascos-González \\ Universidad del Valle. Cali (Colombia) \\ carlos.andres.riascos@correounivalle.edu.co
}

Peter Thomson

Universidad del Valle. Cali (Colombia)

peter.thomson@correounivalle.edu.co

\author{
Shirley Dyke ip \\ Purdue University. West Lafayette, Indiana (United States) \\ sdyke@purdue.edu
}

C. Riascos-González; P. Thomson; S. Dyke "Evaluación del Desempeño de un Amortiguador de Masa Sintonizado no Lineal Mediante Simulaciones Híbridas en Tiempo Real," INGE CUC, vol. 15, no. 2, pp. 11-22, 2019. DOI: http://doi.org/10.17981/ingecuc.15.2.2019.02

\section{Resumen}

Introducción- En este artículo se describe la Simulación Híbrida en Tiempo Real (RTHS) de un Amortiguador no Lineal de Masa Sintonizado (NTMD) y se comparan los resultados con los obtenidos de ensayos experimentales convencionales de una estructura a cortante, de un piso, con el NTMD.

Objetivo- El objetivo de este artículo es valuar la efectividad de una RTHS para estimar el desempeño de un NTMD.

Metodología- La metodología consistió de las siguientes tres etapas: identificación de la estructura principal, diseño del NTMD y evaluación experimental del sistema estructura-NTMD. Para la tercera etapa, se utilizaron RTHS y ensayos sobre mesa vibratoria.

Resultados- Los resultados de los ensayos en mesa vibratoria demostraron que el NTMD redujo el $77 \%$ y $63 \%$ de las aceleraciones pico y RMS de la estructura principal, con respecto a la estructura sin control. Los valores de estas reducciones obtenidos con RTHS fueron $73 \%$ y $63 \%$, respectivamente. Los índices de precisión del sistema de transferencia correspondieron a una amplitud generalizada de 1.01 y un retraso de $2 \mathrm{~ms}$.

Conclusiones- El NTMD, con una razón de masas del 10\%, alcanzó reducciones superiores al $60 \%$ de la respuesta estructural. La RTHS y el ensayo de mesa vibratoria demostraron que el sistema estructura-NTMD tuvo solo un pico en la respuesta en frecuencia. El ruido en la retroalimentación de la RTHS aumentó el grado de amortiguamiento de la estructura controlada. Finalmente, los resultados experimentales demostraron como la RTHS es una técnica que predice efectivamente la aceleración RMS del sistema estructura-NTMD y puede sobreestimar ligeramente su aceleración pico.

Palabras clave- Control estructural; amortiguador no lineal de masa sintonizado; simulación híbrida en tiempo real; mesa vibratoria; interacción amortiguador-estructura.

\begin{abstract}
Introduction- In this paper, the Real-time Hybrid Simulation (RTHS) of a Non-linear Tuned Mass Damper (NTMD) is described, and compares the results with those obtained from conventional experimental tests of a shear, single-storey structure with the NTMD
\end{abstract}

Objective- The objetive of this article is to evaluate the effectiveness of an RTHS in estimating the performance of an NTMD.

Methodology - The methodology consisted of the following three stages: main structure identification, NTMD design, and experimental evaluation of the structure-NTMD system. For the third stage, RTHS and vibrating table tests were used.

Results- The results of the vibrating table tests showed that the NTMD reduced $77 \%$ and $63 \%$ of the peak accelerations and RMS of the main structure, with respect to the structure without control. The values of these reductions obtained with RTHS were $73 \%$ and $63 \%$, respectively. The precision indices of the transfer system corresponded to a generalized amplitude of 1.01 and a delay of $2 \mathrm{~ms}$

Conclusions- The NTMD, with a mass ratio of $10 \%$, achieved reductions of more than $60 \%$ of the structural response. RTHS and the vibrating table test demonstrated that the NTMDstructure system had only one peak in frequency response. The noise in the RTHS feedback increased the degree of damping of the controlled structure. Finally, the experimental results demonstrated how RTHS is a technique that effectively predicts the RMS acceleration of the structure-NTMD system and can slightly overestimate its peak acceleration.

Keywords-Structural control; non-linear tuned mass damper; real-time hybrid simulation; shaking table; damper-structure interaction. 


\section{INTRODUCCIÓN}

El desarrollo de materiales livianos y resistentes aplicados en la Ingeniería Estructural ha permitido la edificación de estructuras flexibles, las cuales son susceptibles a presentar vibraciones excesivas ocasionadas por sismos, cargas eólicas y cargas antrópicas. No obstante, dichas vibraciones pueden ser controladas por Disipadores de Vibración Dinámica (DVA), los cuales logran oponerse a las fuerzas externas mientras transmiten amortiguamiento a la estructura.

Aunque los DVAs han sido ampliamente estudiados durante las últimas cinco décadas, su aceptación ha sido limitada en países en vía de desarrollo tecnológico. La resistencia al uso de estos sistemas es ocasionada por: i) la incertidumbre, ii) los sobrecostos, iii) la desconfianza y iv) la falta de normativa para su implementación [1]. Uno de los dispositivos principales del control estructural es el Amortiguador de Masa Sintonizado (TMD), el cual ha presentado resultados adecuados para el control de estructuras sujetas a cargas eólicas, pero presenta limitaciones para el control de estructuras sometidas a movimiento en la base [2].

Adicionalmente, algunos investigadores han analizado el comportamiento de un Amortiguador de Masa Sintonizado No lineal o NTMD (Non-linear Tuned Mass Damper), a partir de un enfoque numérico, quienes han encontrado importantes variaciones en la respuesta del dispositivo en comparación con el TMD convencional [3], [4]. En esta dirección, sobresalen estudios [5], donde representaron la disipación del NTMD con métodos de amortiguamiento cúbico y amortiguamiento de van der Pol para estimar la disipación de energía del dispositivo. Adicionalmente, el análisis de este sistema de control está aumentando, debido a su versatilidad, como lo evidencian otros estudios [6] con la evaluación de un NTMD en vigas. Otro estudio que resalta la importancia del NTMD en el control estructural [7], el cual con una fuerza de fricción vertical alcanzó un nivel de desempeño equivalente al de múltiples TMDs.

La configuración física del TMD también es un aspecto fundamental para el desempeño del amortiguador. Por ejemplo, el TMD en forma de péndulo ha causado gran interés en el control estructural. Particularmente, este concepto fue adoptado en la implementación del TMD más grande del mundo, el cual se encuentra instalado en el edificio Taipei 101 en Taiwán [8]. Sin embargo, para algunas estructuras, este sistema requiere una gran longitud por efectos de la sintonización. En consecuencia, la configuración de péndulo invertido también ha sido considerada por varios autores, ya que facilita el diseño de los TMDs [9]. No obstante, no se cuenta en la actualidad con evaluaciones experimentales de este tipo de sistema de control estructural, las cuales permitan estimar en función de su razón de masas indicadores como i) reducción pico y RMS de la respuesta de la estructura sin control, ii) grado de amortiguamiento transmitido a la estructura principal y iii) energía disipada por el sistema de control.

Además, el TMD en péndulo invertido presenta de manera intrínseca un comportamiento no lineal en su fuerza cortante. Adicionalmente, el uso de fluido (compresible o incompresible) en su disipador puede agregar un comportamiento no lineal en su fuerza disipativa. Por lo tanto, al responder ambas fuerzas de manera acoplada, se aumenta la complejidad de modelado de dicho sistema. Estas son las razones por las cuales se requieren técnicas especializadas que permitan predecir fielmente la respuesta dinámica de los DVAs modernos; siendo una de estas técnicas las Simulaciones Híbridas en Tiempo Real o RTHS (Real-Time Hybrid Simulations).

Las RTHS son una técnica moderna con un enfoque físico y cibernético utilizado para la evaluación experimental de sistemas complejos, como los NTMDs. Estas simulaciones consideran las partes de una estructura con comportamiento predecible como subestructura numérica y los componentes difíciles de modelar como subestructura experimental [10]-[12]. Debido a lo anterior, las RTHS permiten a los investigadores evaluar las partes críticas de las estructuras con una inversión eficiente de recursos financieros, tiempo y espacio [13]. Esto ha permitido a las RTHS ser consideradas como una técnica factible para la evaluación de sistemas de control estructural.

La evaluación de la respuesta dinámica de un amortiguador viscoso [14] ha permitido encontrar una correspondencia adecuada entre los resultados experimentales y los simulados. No obstante [15], con la implementación de una RTHS se permite estimar la respuesta de una estructura a cortante, con bajo nivel de amortiguamiento. Otras aplicaciones de RTHS en dispositivos de control han considerado el amortiguador de líquido sintonizado, amortiguador de columna liquida sintonizada y amortiguador magnetoreológico [16]-[18]. Y sin embargo, no se ha aplicado RTHS para estudiar el comportamiento de un NTMD en forma de péndulo invertido.

En este artículo se describe la evaluación experimental de un NTMD en forma de péndulo invertido, con fluido compresible en su disipador. Dicho análisis se realiza con ensayos de mesa vibratoria y RTHS, con lo cual, se evalúa el desempeño del novedoso y practico sistema de control ante movimiento en la base. Adicionalmente, se estudió la precisión de la RTHS para estimar el comportamiento del NTMD, haciendo especial énfasis en el seguimiento del sistema de transferencia. 
Finalmente, se puede confirmar como las RTHS son una técnica adecuada para la evaluación de sistemas de rehabilitación estructural no convencionales, al ser una necesidad apremiante en países en vía de desarrollo tecnológico, como se describe en las conclusiones de este artículo.

\section{Estado DEL ARte}

\section{A. Evaluación dinámica de sistemas de control}

Actualmente, existen tres métodos para evaluar el comportamiento de estructuras sujetas a cargas, los cuales son: las pruebas cuasi estáticas, los ensayos de mesa vibratoria y las simulaciones híbridas en tiempo real [19].

Los ensayos en mesa vibratoria son una de las técnicas más precisas y confiables para el estudio de estructuras sometidas a cargas externas. Sin embargo, dependiendo de la capacidad de la mesa vibratoria, estos ensayos tienen limitaciones en el sistema a evaluar, con respecto a su tamaño, peso o rigidez [20].

A diferencia de los ensayos en mesa vibratoria, en la implementación de una RTHS solo se debe construir la parte crítica de la estructura compleja, y el resto de la estructura es representado de manera numérica [21]. La Fig. 1 muestra un diagrama general de una RTHS, el cual incluye una subestructura experimental, un bloque de integración de la subestructura numérica y un sistema de transferencia. En este diagrama, $x_{c}$ representa el desplazamiento calculado, $x_{r}$ el desplazamiento real, $x_{m}$ el desplazamiento medido y $F_{m}$ la fuerza medida. Los bloques A/D y D/A hacen referencia a convertidores de señal analógica a digital y de señal digital a analógica, respectivamente.

Uno de los desafíos principales de la RTHS es la generación de las condiciones de frontera en la subestructura física, la cual requiere de un conocimiento detallado de la dinámica del actuador y una estimación del efecto que tengan los componentes físicos sobre el sistema de transferencia [22].
En las diferentes fases de la RTHS se presentan retrasos, inherentes a los procesos de comunicación, comprometiendo la estabilidad y el desempeño de las RTHS. Con el objetivo de disminuir el efecto de dichos retrasos [23] se utiliza el método de integración modificado de Runge-Kutta (MRK). Mientras [24] agregaron a las ventajas de un controlar de norma $H_{\infty}$ las características de un Estimador Cuadrático Lineal (LQE) y un lazo de realimentación directa. Esta combinación denominada RIAC (Robust Integrated Actuator Control) que reduce los efectos desfavorables del ruido en la medición y del retraso (delay) de la simulación.

Con el objetivo de mejorar la confiabilidad de la información obtenida de la subestructura experimental, durante una RTHS [24], se combinaron la RTHS con algoritmos de actualización de modelos o RTHSMU (del inglés Real Time Hybrid Simulation with Model Updating Algorithm). Este novedoso método mejora considerablemente la evaluación de la subestructura experimental, especialmente cuando el modelo de la subestructura numérica carece de precisión.

Actualmente, investigadores están usando cada vez más las RTHS, y se está convirtiendo en una técnica determinante para la evaluación de sistemas de control. Por ejemplo, [25] evaluaron el desempeño de un amortiguador magnetoreológico (MR) a escala completa a través de las RTHS. Además, estudiaron el efecto de un amortiguador de líquido sintonizado (TLD: Tuned Liquid Damper) utilizando RTHS [26]. Adicionalmente, estos investigadores evaluaron el sistema estructura-amortiguador utilizando ensayos de mesa vibratoria. Al comparar los resultados obtenidos por ambos métodos, dichos investigadores encontraron una alta precisión de la RTHS en la predicción del comportamiento dinámico de la estructura controlada.

Analizaron la efectividad de un amortiguador de columna líquida sintonizado con presión (PTLCD) para controlar la respuesta de un sistema de un grado de libertad [26]. También, estos investigadores utilizaron índices de evaluación para RTHS, y reportaron una buena precisión de la simulación desarrollada.

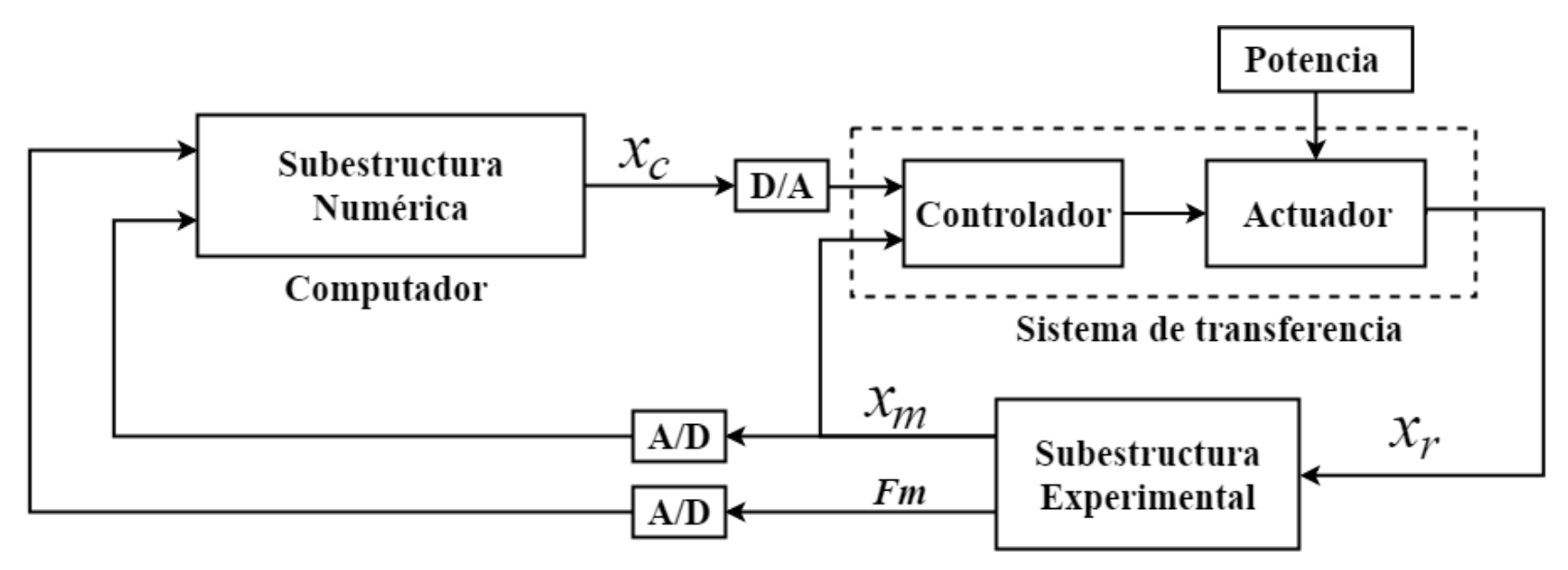

Fig. 1. Diagrama de bloques de una RTHS.

Fuente: Modificado de [27]. 
Recientemente, evaluaron el desempeño de un amortiguador sintonizado de columna líquida en una estructura de referencia de 9 pisos [17]. Estos investigadores incluyeron efectos de interacción suelo-estructura en la simulación, lo cual muestra la versatilidad que presentan las RTHS para evaluar sistemas no lineales.

\section{B. Indices de precisión para RTHS}

Algunos índices de evaluación de respuesta han sido propuestos en la literatura para determinar la precisión del sistema de transferencia de la RTHS, los cuales pueden ser clasificados en índices locales y globales [28]. Los primeros se enfocan en la capacidad de seguimiento del sistema de transferencia, mientras los segundos consideran la interacción entre las subestructuras numérica y experimental. Ambos grupos de índices incluyen evaluaciones en los dominios del tiempo y de la frecuencia.

Entre los índices de evaluación local se encuentra el índice de evaluación en frecuencia (FEI: frequency evaluation index), el cual compara los desplazamientos calculado $\left(x_{c}\right)$ y medido $\left(x_{m}\right)$ del sistema de transferencia, utilizando la Transformada Rápida de Fourier (FFT). Las FFTs de dichas señales se muestran como $y_{c}$ y $y_{m}(1)$, respectivamente, donde \|\| representa la norma de cada número complejo del vector correspondiente. En dicha ecuación la potencia $l$ toma un valor de 2 y $N$ es la longitud de la señal a evaluar.

A partir del FEI, se pueden calcular los índices amplitud $A_{o}$ y ángulo $(\varnothing)$ generalizados de la RTHS. El primero indica el nivel de correspondencia en amplitud entre $x_{c}$ y $x_{m}$. Por lo cual, su valor ideal es igual a uno (1.0). Adicionalmente, el delay del sistema de transferencia puede ser determinado a partir de (Ø) (3). En la evaluación del seguimiento del sistema de transferencia, también es determinante conocer la frecuencia que tiene mayor participación durante la RTHS. Este parámetro es la frecuencia equivalente $\left(f^{\mathrm{eq}}\right)$, la cual se obtiene (4), donde $f_{i}$ es el vector de frecuencias.

El índice de error pico es definido como índice global [28], el cual se encarga de determinar la mayor diferencia ocurrida entre los desplazamientos calculado y medido (6). Otro indicador, definido en el dominio del tiempo, para establecer la confiabilidad de la RTHS es el error RMS (Root Mean Square) normalizado (NRMS error) (7). En la literatura, algunos parámetros similares a estos son considerados admisibles si presentan valores inferiores al 20\% [27].

El comportamiento de una RTHS también se puede evaluar, de manera global, con el error de energía $E_{\text {err }}$. Este índice se define como la diferencia entre la energía obtenida con el desplazamiento calculado y la obtenida con el desplazamiento medido (8)-(10). Ambas energías son evaluadas ante la fuerza medida de la subestructura experimental.

$$
\begin{gathered}
F E I=\sum_{j=1}^{N}\left\{\frac{y_{m}(j)}{y_{c}(j)} \cdot \frac{\left\|y_{c}(j)\right\|^{l}}{\sum_{i=1}^{N}\left\|y_{c}(i)\right\|^{l}}\right\} \\
A_{0}=\|F E I\| \\
\varnothing=\arctan [\operatorname{Im}(F E I) / R e(F E I)] \\
\delta=-\frac{\emptyset}{2 \pi f^{e q}} \\
f^{e q}=\frac{\sum_{j=1}^{N}\left\{\left\|y_{c}(j)\right\|^{l} \cdot f_{j}\right\}}{\sum_{j=1}^{N}\left\|y_{c}(j)\right\|^{l}} \\
e_{p}=\frac{\|\left. x_{c}\right|^{\max }-\left|x_{m}\right|^{\max \mid}}{\left|x_{m}\right|^{\max }} \\
\text { NRMS error }=\sqrt{\frac{\sum_{i=1}^{N}\left[x_{m}(i)-x_{c}(i)\right]^{2}}{\sum_{i=1}^{N} x_{c}(i)^{2}}} \\
E_{\text {err }}=E_{c}-E_{m} \\
\mathrm{E}_{c}=\int F_{m} d x_{c} \\
\mathrm{E}_{m}=\int F_{m} d x_{m}
\end{gathered}
$$

\section{Amortiguadores de masa sintonizado}

En este artículo se evalúa la efectividad de los ensayos híbridos para una estructura con un Amortiguador de Masa Sintonizado o TMD (Tuned Mass Damper). Los TMDs han sido ampliamente analizados por investigadores, con el fin de evaluar su desempeño ante sismos, cargas eólicas y antrópicas. Este sistema es la segunda tecnología de control más implementada a nivel mundial, después de los aisladores sísmicos. El TMD implementado en el edificio Taipei 101 [29], es el TMD más grande del mundo, con un peso de 660 toneladas.

El TMD fue patentado en 1909 por Frahm [30] y desde esta fecha, se han reportado numerosas adaptaciones, con respecto a la versión original. Por ejemplo, desarrollaron un TMD Bidireccional y Homogéneo (BH-TMD) [31], el cual puede ser sintonizado en dos direcciones ortogonales a frecuencias diferentes. Este sistema fue evaluado experimentalmente y alcanzó reducciones hasta del 60\% del desplazamiento estructural, con respecto a la estructura sin control. Otros [32] analizaron el efecto de un TMD, cuya disipación fue proporcionada por un amortiguador magnetoreológico. Y después utilizaron un Amortiguador de Masa Sintonizado con Longitud de Péndulo Adaptable o (APTMD) [3].

\section{Metodología}

El desarrollo de una simulación híbrida en tiempo real para la evaluación experimental de un NTMD requirió una caracterización detallada de la estructura principal. A partir de dichas características, se diseñó 
el dispositivo de control no lineal. Una vez construido este dispositivo, se aplicaron dos técnicas de evaluación dinámica. La primera y convencional denominada ensayos en mesa vibratoria, y la moderna y eficiente técnica de simulaciones híbridas en tiempo real. Los resultados de esta última fueron evaluados con índices de precisión, propuestos en la literatura, y comparados con los obtenidos de los ensayos de mesa vibratoria, como se describe en las siguientes secciones.

\section{A. Estructura principal}

La estructura principal es un pórtico a cortante, el cual consta de dos láminas de acero y dos láminas de acrílico, como se muestra en la Fig. 2. Esta estructura tiene una masa de $1 \mathrm{~kg}$, una frecuencia natural de $3.6 \mathrm{~Hz}$ y una razón de amortiguamiento de $1 \%$. La frecuencia y el amortiguamiento de la estructura fueron obtenidos a partir de ensayos de vibración libre y forzada, donde los métodos de decremento logarítmico y ancho de banda de media potencia fueron empleados, respectivamente.
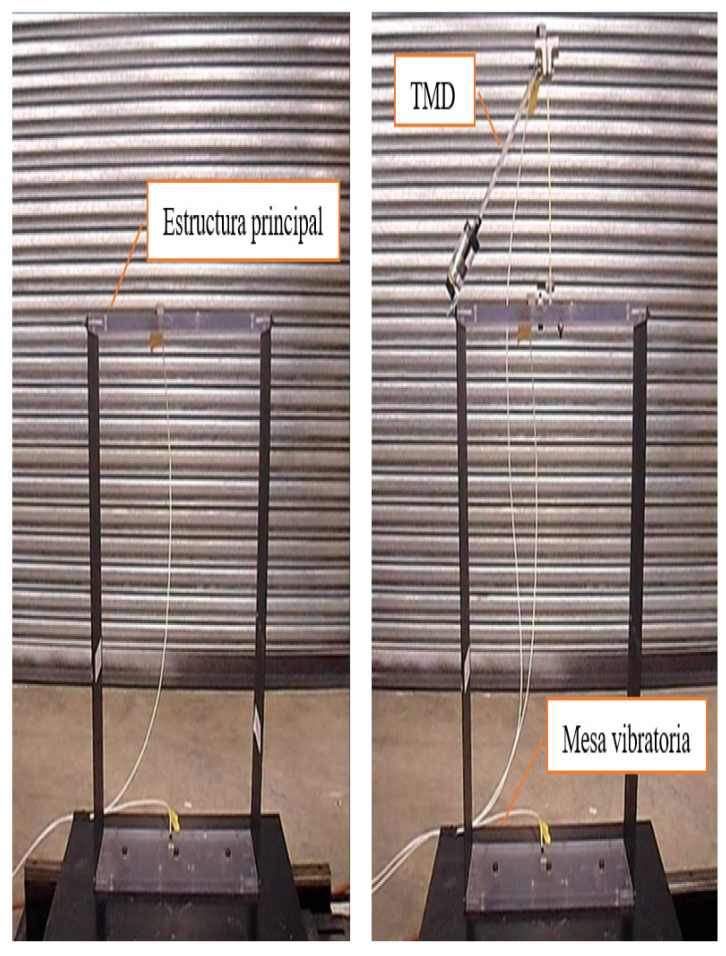

Fig. 2. Estructura principal.

Fuente: Autores.

El ensayo de vibración forzada de la estructura consistió en un movimiento en la base, el cual fue descrito por un barrido de frecuencias entre 1 y $5 \mathrm{~Hz}$, con una duración de 240 segundos y una amplitud de $1 \mathrm{~mm}$. A partir de los resultados de este ensayo, se calculó una función de respuesta en frecuencia (FRF) de la estructura, donde la señal de entrada corresponde a la fuerza efectiva producida por el movimiento en la base y la señal de salida es la aceleración estructural. Esta FRF fue calculada a través de la función de Matlab tfestimate. Luego, una función de transferencia fue obtenida para representar analíticamente la FRF. Esta representación fue calculada empleando un ajuste por mínimos cuadrados, en el dominio de la frecuencia y sirvió como modelo analítico de la estructura principal.
La función de transferencia obtenida (11), es donde $F(s)$ es la transformada de Laplace de la fuerza efectiva y $A(s)$ es la transformada de Laplace de la aceleración estructural. A partir de la función obtenida, se calculó la respuesta simulada ante el barrido de frecuencias descrito previamente. Esta señal simulada fue comparada con la salida experimental (12), lo cual reportó un ajuste del 82\%. En dicha expresión $y_{\text {exp }} \mathrm{y} y_{\text {sim }}$ son las salidas experimental y simulada, respectivamente. El valor medio de $y_{\exp }$ se muestra como $\bar{y}_{\exp } \mathrm{y}\|\|$ indica el cálculo de la norma-2. La Fig. 3 muestra las funciones de transferencia experimental y ajustada de la estructura principal, donde se puede observar la correspondencia de ambas representaciones.

$$
\begin{aligned}
& G s \quad \frac{A(s)}{F s} \frac{-589.1}{s^{2}} \frac{s}{f i t=100\left(1-\frac{\left\|y_{\text {exp }}-y_{\text {sim }}\right\|}{\left\|y_{\text {exp }}-\bar{y}_{\text {exp }}\right\|}\right)}
\end{aligned}
$$

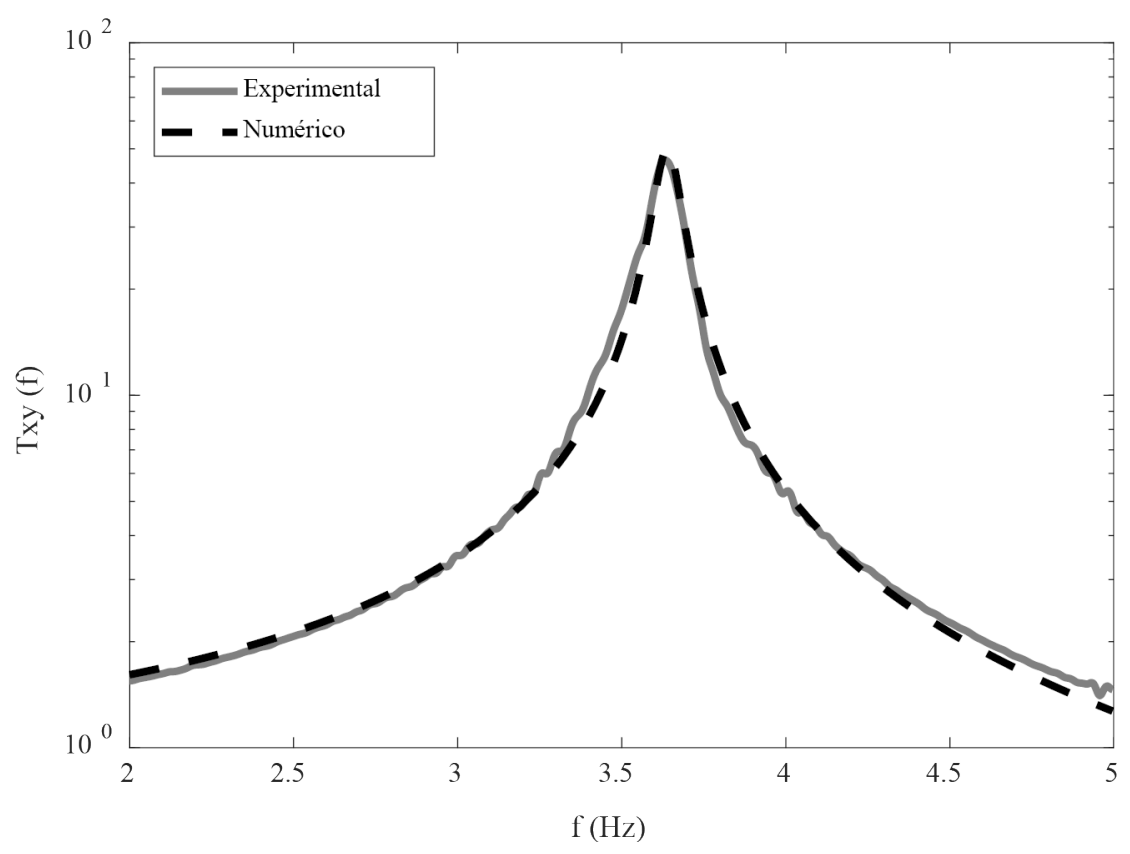

Fig. 3. Comparación entre la función de transferencia ajustada y la FRF.

Fuente: Autores.

\section{B. Amortiguador no lineal de masa sintonizado}

El amortiguador no lineal de masa sintonizado (NTMD: Non-linear Tuned Mass Damper) usado en este trabajo, presenta la facilidad de implementación, típica del TMD convencional, mas agrega características favorables con respecto a la disipación.

El NTMD consistió en una masa fija en la parte superior, en forma de péndulo invertido; una columna flexible y un disipador. La masa fija incluyó dos ángulos de aluminio de $2.5 \mathrm{~cm}$ por lado con $5 \mathrm{~mm}$ de espesor. El disipador empleado en el NTMD está compuesto por un cilindro de $8 \mathrm{~mm}$ de radio y $4 \mathrm{~cm}$ de longitud, el cual hace pasar aire a través de un orificio regulable, ubicado en uno de sus extremos. El disipador estaba conectado a la masa del NTMD, como se muestra en la Fig. 4. 


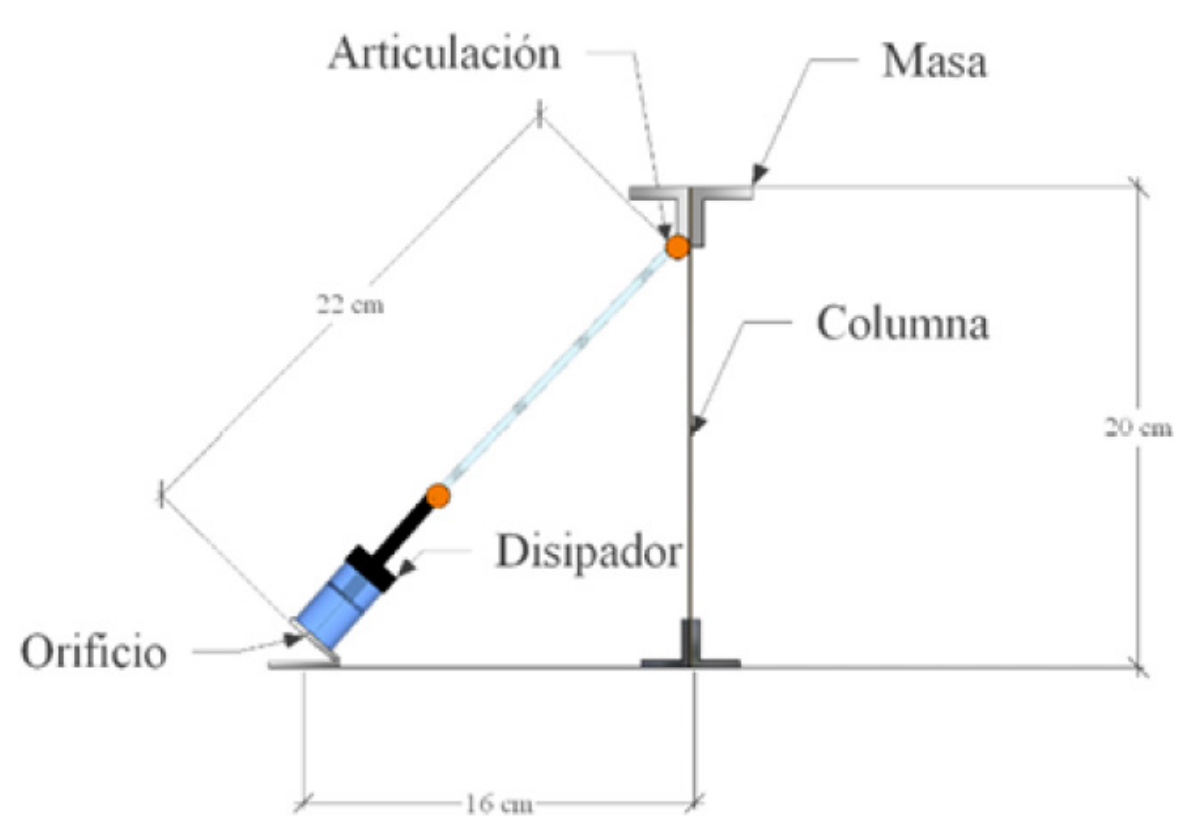

Fig. 4. NTMD.

Fuente: Autores.

La columna flexible del NTMD fue modelada como un elemento sin masa, empotrada en su base. Por lo tanto, la longitud de la barra $(L)$ fue calculada en términos de la frecuencia deseada $\left(f_{d}\right)$ en el NTMD, la masa concentrada $\left(m_{d}\right)$ en la parte superior del mismo y las propiedades mecánicas del elemento (15), donde, $I$ es el momento de inercia y $E$ es el módulo de elasticidad.

$$
L=\sqrt[3]{\frac{3 E I}{m_{d}\left(2 \pi f_{d}\right)^{2}}}
$$

Para el diseño de la configuración nominal del NTMD se utilizaron las razones óptimas de sintonización y amortiguamiento de un TMD clásico. La razón de sintonización hace referencia a la relación entre la frecuencia del amortiguador y la frecuencia de la estructura principal, mientras la razón de amortiguamiento se define como la relación entre el amortiguamiento del sistema y su amortiguamiento crítico [33].

Las propiedades óptimas del TMD son generalmente funciones de la razón entre la masa del amortiguador y la de la estructura $(\mu)$. Estas expresiones cambian dependiendo del comportamiento de la excitación (armónico o aleatorio), del tipo de excitación (fuerza o aceleración en la base) y del tipo de respuesta del sistema (desplazamiento o aceleración) que se desea minimizar [34]. En este estudio se utilizaron las expresiones correspondientes a minimización del desplazamiento estructural para una fuerza armónica (14)-(15). Con una razón de masas del 10\%, las razones óptimas de sintonización y amortiguamiento del TMD son 91\% y $19 \%$, respectivamente.

$$
\begin{gathered}
\gamma_{o p t}=\frac{1}{1+\mu} \\
\zeta_{o p t}=\sqrt{\frac{3}{8}\left(\frac{\mu}{1+\mu}\right)}
\end{gathered}
$$

A partir de la frecuencia natural de la estructura principal y la razón de sintonización óptima calculada previamente, se obtuvo una frecuencia natural de $3.3 \mathrm{~Hz}$ para el NTMD. La masa concentrada de este sistema fue $0.1 \mathrm{~kg}$, de acuerdo con la razón de masas seleccionada. El módulo de elasticidad de la columna fue de 110GPa, ya que se utilizó un elemento de cobre, y el área de la sección transversal fue 1 x $5 \mathrm{~mm}^{2}$. De esta manera, la longitud requerida de la columna del NTMD era de $200 \mathrm{~mm}$.

\section{Evaluación en mesa vibratoria.}

La Fig. 5 muestra la configuración del sistema estructura-NTMD, el cual se instaló sobre una mesa vibratoria uniaxial. Este sistema fue sometido a un barrido de frecuencias entre 1 y $5 \mathrm{~Hz}$, con duración de 240 segundos y amplitud de $1 \mathrm{~mm}$ para medir el desempeño del NTMD, al compararlo con la respuesta de la estructura sin control.

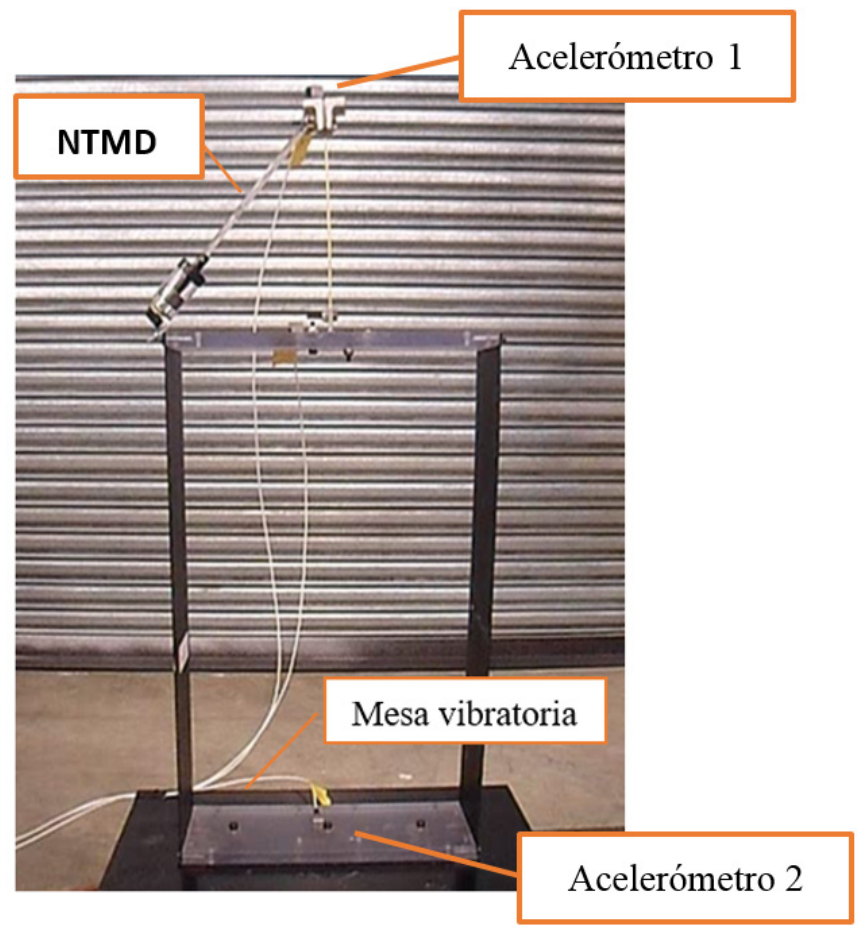

Fig. 5. Configuración del sistema estructura-NTMD.

Fuente: Autores.

Las ecuaciones (16)-(17) representan el movimiento de los sistemas estructura principal y estructura-NT$\mathrm{MD}$, respectivamente. En estas ecuaciones $m, c$ y $k$ son los parámetros de masa, amortiguamiento y rigidez de la estructura principal, mientras que $m_{d}, c_{d}$ y $k_{d}$ son los parámetros equivalentes para el NTMD. Adicionalmente, $u$ y $u_{d}$ representan los desplazamientos de la estructura y del NTMD, con respecto a la base, $\ddot{u}_{g}$ es la aceleración en la base inducida por la mesa vibratoria y $\left[g_{1}\left(\stackrel{i}{u}, \stackrel{\circ}{u}_{d}, t\right) g_{2}\left(\stackrel{\circ}{u}, \stackrel{\circ}{u}_{d}, t\right)\right]^{T}$ es un vector que contiene funciones relacionadas con la disipación de la estructura y la del amortiguador.

$$
\begin{gathered}
m \ddot{u}+c \dot{u}+k u=-m \ddot{u}_{g} \\
{\left[\begin{array}{cc}
m & 0 \\
0 & m_{d}
\end{array}\right]\left\{\begin{array}{l}
\ddot{u} \\
\ddot{u}_{d}
\end{array}\right\}+\left[\begin{array}{cc}
k+k_{d} & -k_{d} \\
-k_{d} & k_{d}
\end{array}\right]\left\{\begin{array}{c}
u \\
u_{d}
\end{array}\right\}} \\
=\left\{\begin{array}{c}
g_{1}\left(\dot{u}, \dot{u}_{d}, t\right) \\
g_{2}(u, u
\end{array}\right\}-\left\{\begin{array}{c}
m \\
m_{d}, t
\end{array}\right\} \ddot{u}_{g}
\end{gathered}
$$


En el ensayo de mesa vibratoria, se instalaron dos acelerómetros microelectromecánicos o MEMS por sus siglas en inglés. Uno de ellos fue instalado sobre la estructura principal y el otro sobre el NTMD, como se muestra en al Fig. 5. Dichos sensores registraron la respuesta en aceleración de la estructura con control, la cual fue generada por la deformación a cortante inducida por la excitación en la base.

\section{Simulación híbrida en tiempo real}

El sistema estructura-NTMD también fue evaluado con una simulación híbrida en tiempo real, donde la estructura principal fue considerada como la subestructura numérica (SN) y el NTMD como la subestructura experimental (SE). La ecuación (18) representa la RTHS desde el punto de vista de la $\mathrm{SN}$, siendo R(ud, $\left.\stackrel{\circ}{u}_{d}, u, \stackrel{u}{u}, \ddot{u}_{g}, t\right)$ la fuerza resultante inducida por la SE. La expresión de esta fuerza fue reorganizada para ser función de la aceleración total de la masa del NTMD (19). De esta manera, $R\left(\ddot{u}_{d}^{T}\right)$ determina exactamente la fuerza de control del NTMD, incluyendo: i) la no linealidad debido al flujo de aire en el disipador, ii) la fricción entre el pistón y el cilindro del disipador y iii) eventuales choques entre el pistón y los extremos del cilindro.

$$
\begin{gathered}
m \ddot{u}+c \dot{u}+k u=-m \ddot{u}_{g}+R\left(u_{d}, \dot{u}_{d}, u, \dot{u}, t\right) \\
R\left(\ddot{u}_{d}^{T}\right)=-m_{d}\left(\ddot{u}_{g}+\ddot{u}_{d}\right)=-m_{d} \ddot{u}_{d}^{T}
\end{gathered}
$$

Esta RTHS fue programada en Simulink y ejecutada con un procesador SpeedGoat. Para lo cual, se utilizó un computador anfitrión con el protocolo Xpc de Matlab y una frecuencia de muestreo de $1024 \mathrm{~Hz}$. El SpeedGoat tiene alta capacidad de procesamiento para garantizar la evaluación en tiempo real de la simulación híbrida. La retroalimentación de la fuerza del NTMD se calculó a partir de la aceleración medida $\left(\ddot{u}_{d}^{T}\right)(19)$. En este caso el acelerómetro 2 fue instalado sobre la mesa vibratoria, la cual reprodujo la deformación a cortante de la subestructura numérica.
La Fig. 7 presenta un esquema de la RTHS realizada, cuyos componentes se describirán a continuación:

Inicialmente, se estableció la entrada al sistema que en este caso fue la fuerza efectiva ocasionada por una aceleración en la base, $a_{g}(t)$. Dicho movimiento fue descrito por un barrido de frecuencias entre 1 y $5 \mathrm{~Hz}$, con duración de 240 segundos y amplitud de $1 \mathrm{~mm}$. Esta señal fue enviada al modelo de la subestructura numérica, la cual se le adicionó la masa fija del NTMD. Luego, el desplazamiento de la SN fue tomado como la señal de referencia del sistema de transferencia, $x_{c}$, el cual fue modificado por un compensador de retraso. La aceleración de la SN fue representada por $a(t)$.

En esta RTHS, se utilizó una mesa vibratoria para generar el desplazamiento en la subestructura experimental. Este sistema fue guiado por un controlador $H_{\infty}$. Para mejorar el seguimiento del sistema de transferencia se estimaron los estados de la mesa, $x_{e s t}$, empleando un filtro de Kalman.

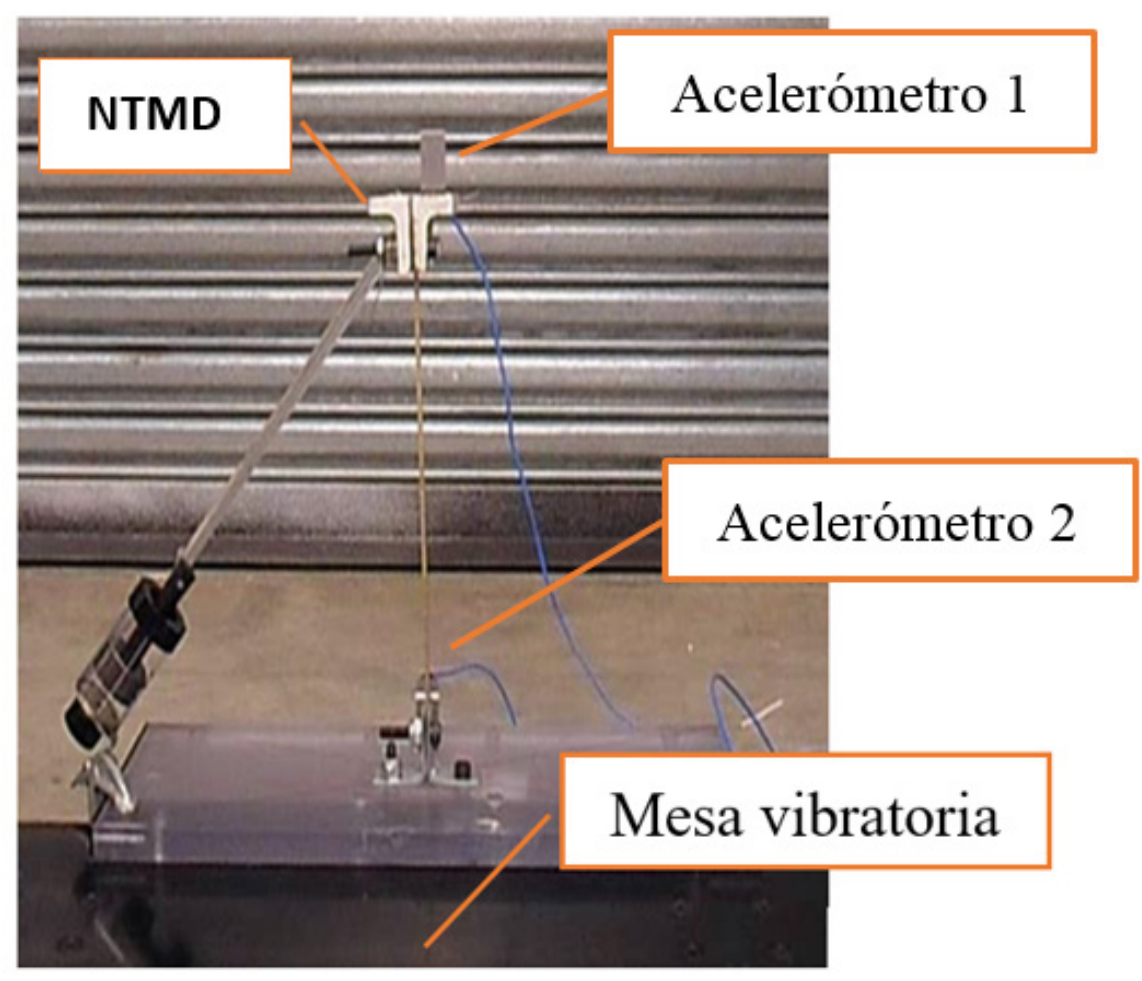

Fig. 6. Configuración de la SE. Fuente: Autores

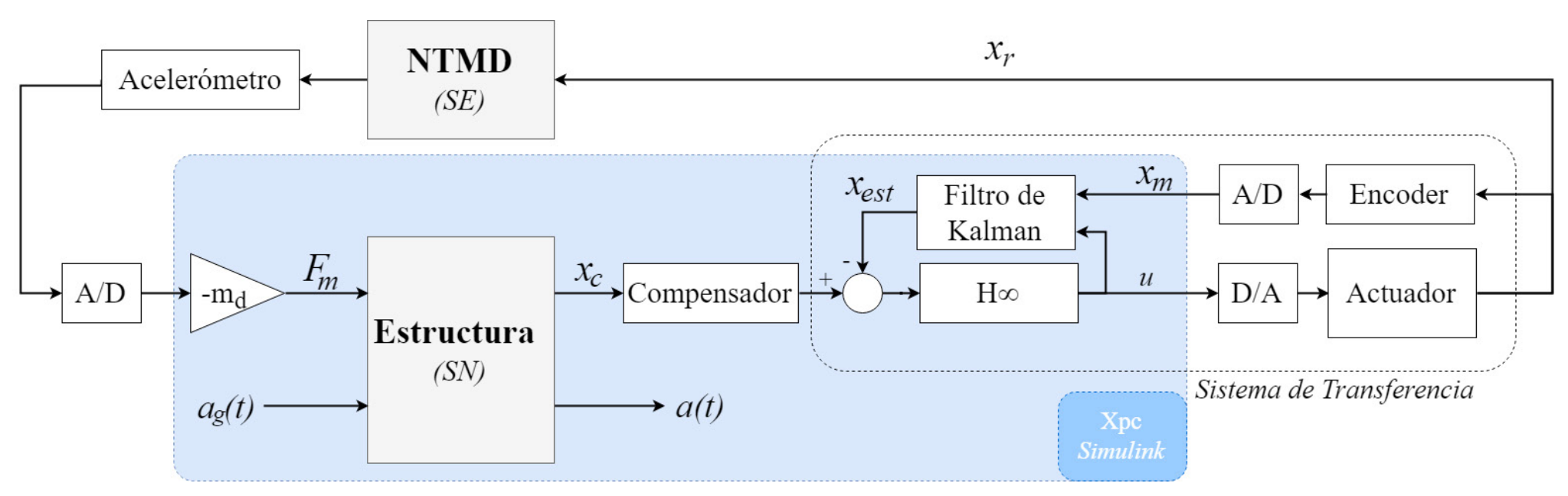

Fig. 7. Configuración de la RTHS.

Fuente: Autores. 
El desplazamiento de la SN fue transferido a la SE (Fig. 6). La SE respondió a dicho movimiento y su fuerza de reacción fue calculada para cerrar el lazo de la RTHS. El desplazamiento del sistema de transferencia $x_{m}$ fue medido a partir de un encoder incorporado en actuador de la mesa, el cual fue comparado con el desplazamiento calculado de la RTHS $x_{c}$, como se detalla en siguientes secciones.

\section{Resultados}

\section{A. Desempeño del NTMD obtenido por ensayo de mesa vibratoria}

La Fig. 8 muestra la respuesta, en el dominio del tiempo y en el domino de la frecuencia, de la estructura sin control y de la estructura controlada por el NTMD, ambas ante un barrido de frecuencias descrito en la Sección III. De esta evaluación, se determinó que el amortiguador alcanzó reducciones del 77\% y del $63 \%$ de la respuesta pico y RMS, con respecto a la estructura sin control. Adicionalmente, se encontró que la estructura controlada tiene una frecuencia natural menor que la presentada por la estructura sin control. Esto fue ocasionado por el incremento de la masa generado por el NTMD sobre la estructura principal. Adicionalmente, se encontró que el NTMD aumentó 6.7 veces la razón de amortiguamiento de la estructura principal, como lo indica el método de ancho de banda evaluado en las funciones de transferencia de la Fig. 8. Particularmente, la respuesta en frecuencia del sistema estructura-NTMD no presentó los dos picos típicos de un sistema de un grado de libertad controlado por un TMD clásico. Esto fue generado por la no linealidad del amortiguamiento del NTMD [4].

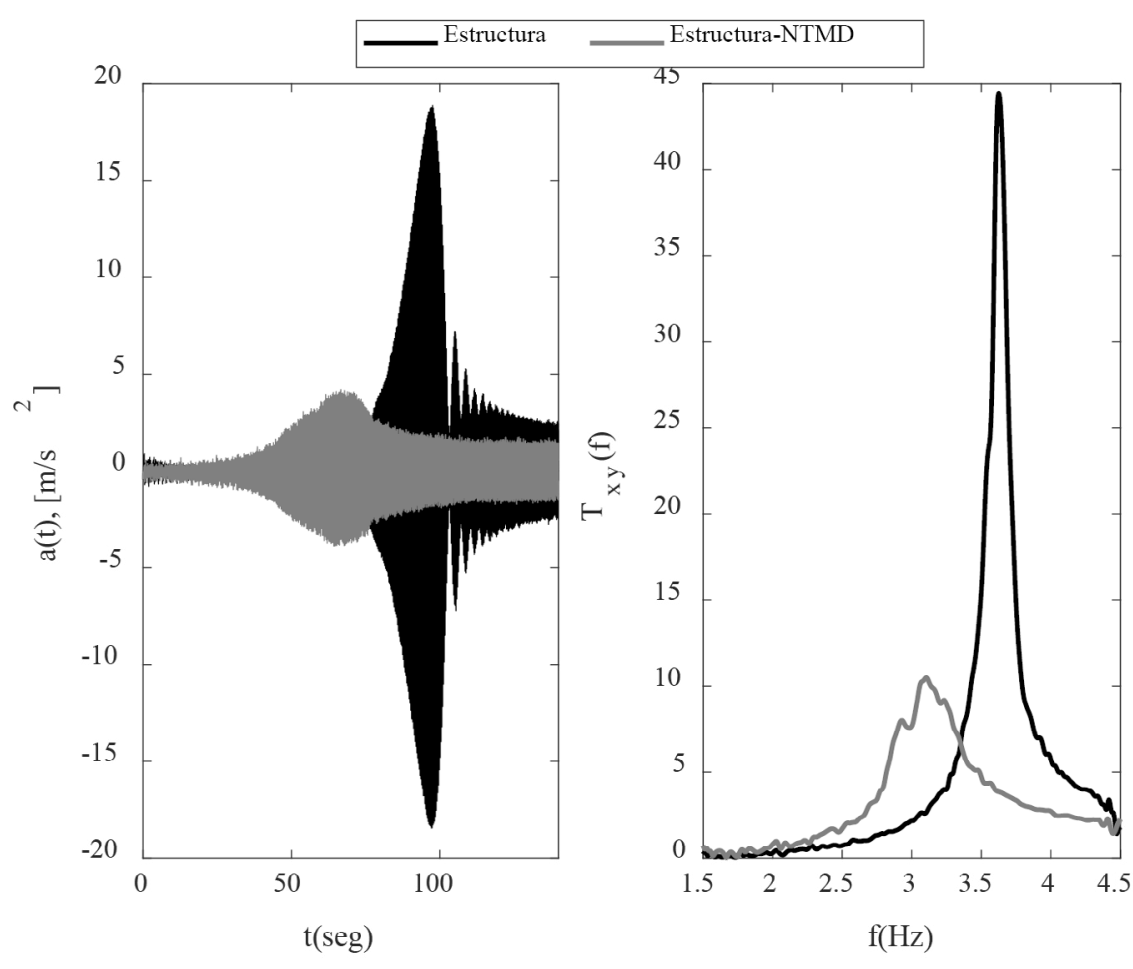

Fig. 8. Respuesta de la estructura principal y del sistema estructura-NTMD con ensayo de mesa vibratoria.

Fuente: Autores.

\section{B. Desempeño del NTMD obtenido con RTHS}

La respuesta en aceleración de la subestructura numérica de la RTHS (estructura principal), se muestra en el dominio del tiempo y en el dominio de la frecuencia en la Fig. 9. A partir de lo cual, se encontró que la subestructura experimental alcanzó reducciones del 73\% y 63\% de la respuesta pico y RMS, con respecto a la respuesta de la estructura principal sin control, medida con ensayos de mesa vibratoria. El NTMD, evaluado con la RTHS, aumentó 10.5 veces la razón de amortiguamiento de la estructura principal. Nótese que este sistema presentó una frecuencia fundamental de $3.2 \mathrm{~Hz}$, debido a la masa del NTMD, mientras que la frecuencia de la estructura sin control fue de $3.6 \mathrm{~Hz}$. Los resultados obtenidos con esta técnica tampoco presentaron los picos típicos de un sistema controlado por un TMD lineal, al igual que los ensayos en mesa vibratoria.

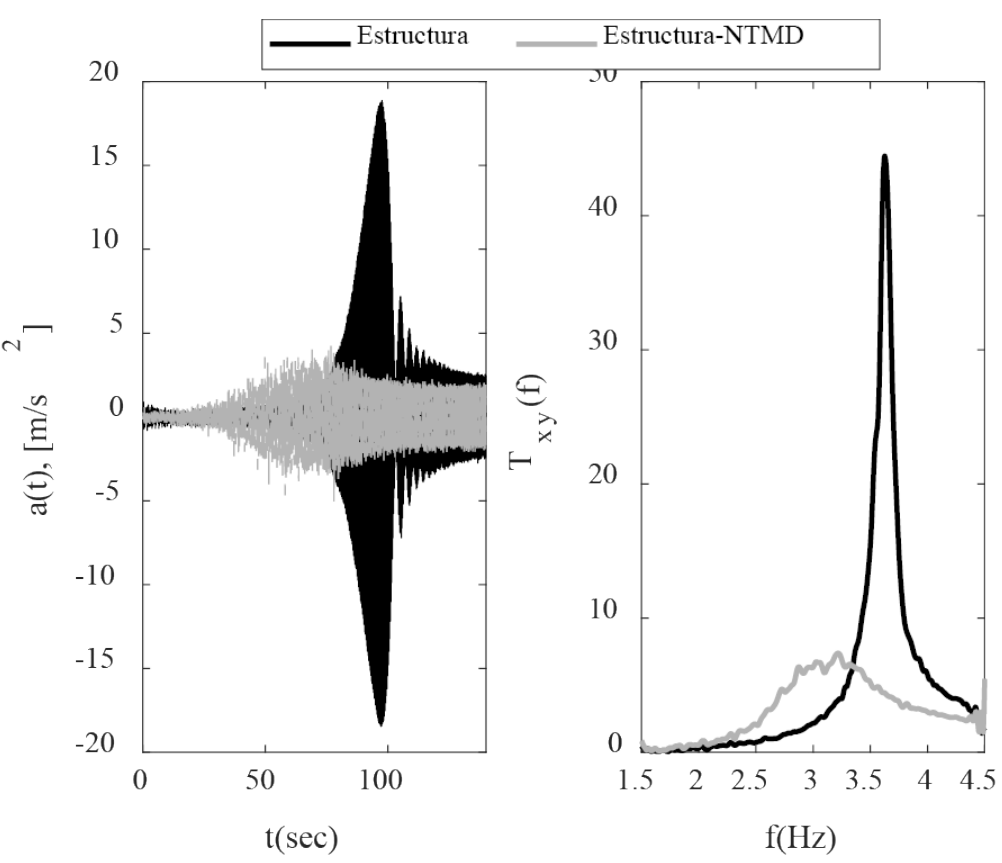

Fig. 9. Respuesta de la estructura principal y del sistema estructura-NTMD con RTHS.

Fuente: Autores.

\section{Evaluación del Sistema de Transferencia de la RTHS}

Una de las fases más importantes de las RTHS es el seguimiento del sistema de transferencia, por lo cual el comportamiento de la mesa vibratoria se evaluó a partir del grupo de índices de precisión descritos en la sección 1-B, demostrando como el sistema de transferencia presenta un gran desempeño. A partir del FEI, se encuentra una amplitud generalizada de $1.01 \mathrm{y}$ un retraso del sistema de transferencia de tan solo dos periodos de muestreo (2 ms), como se muestra en la Tabla 1.

TABLA 1. INDICADORES DE EVALUACIÓN

\begin{tabular}{|c|c|c|c|}
\hline$F E I$ & $\mathrm{~A}_{0}$ & $\theta\left(^{\circ}\right)$ & $(\mathrm{ms})$ \\
\hline $1.01+0.04 i$ & 1.01 & -2 & 2 \\
\hline$N R M S_{\text {error }}(\%)$ & $e_{p}(\%)$ & $f^{e q}(H z)$ & $E_{\text {err }}(\%)$ \\
\hline 7.5 & 15 & 3.0 & $8 \%$ \\
\hline
\end{tabular}

Fuente: Autores. 
La Fig. 10 muestra la orientación obtenida del FEI, donde se puede observar que es muy cercana a la orientación de un FEI ideal, con amplitud generalizada de 1.0 y ángulo generalizado de $0^{\circ}$, lo cual determina que el sistema de transferencia presentó un alto nivel de seguimiento. Por otro lado, la frecuencia equivalente de la RTHS fue $3 \mathrm{~Hz}$ y dicho parámetro se aproxima a la frecuencia natural del sistema estructura-NTMD. Además, tanto el error pico como el error NRMS fueron menores que el umbral del 20\%. Esto indica que el sistema de transferencia reprodujo adecuadamente la respuesta de la subestructura numérica.

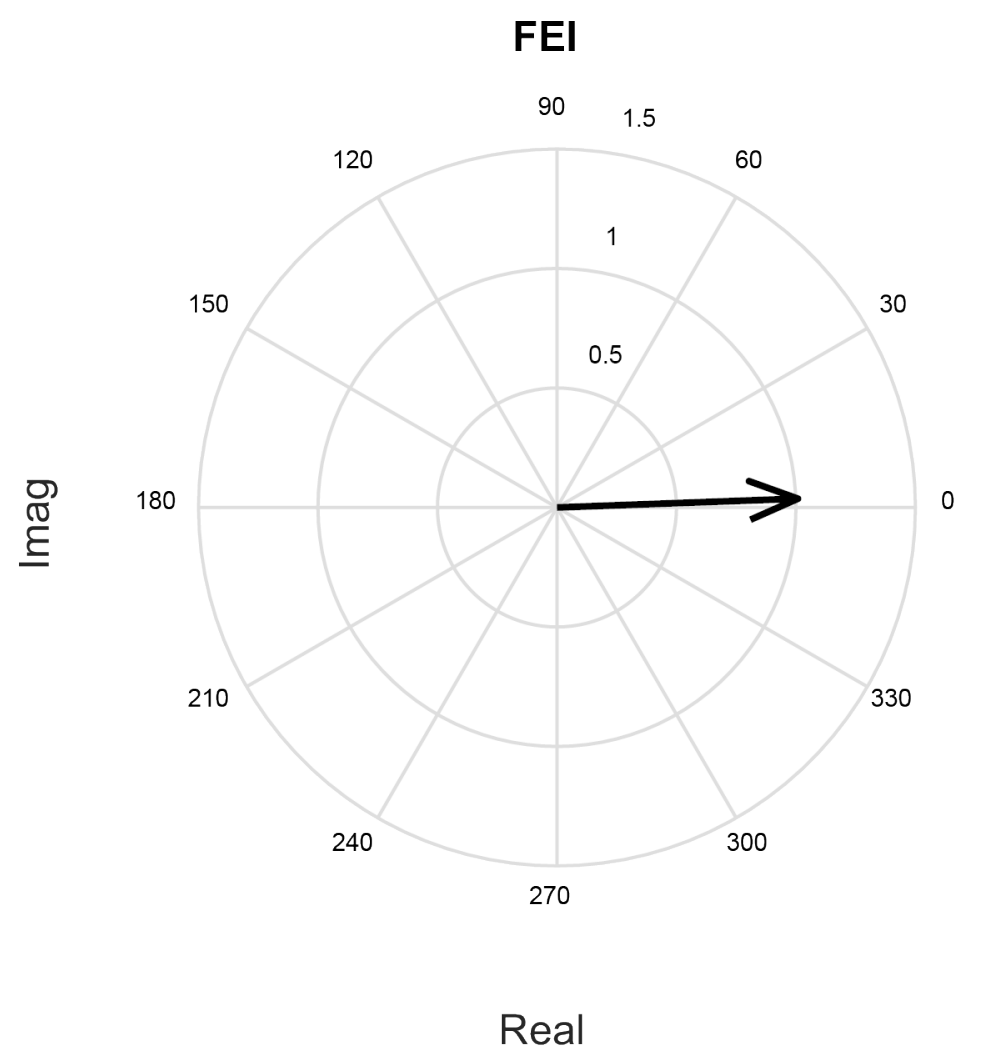

Fig. 10. FEI.

Fuente: Autores.

Adicionalmente, se evaluaron las energías calculada y medida del sistema de transferencia. La primera se obtuvo en términos de la fuerza medida y el desplazamiento calculado, mientras que la segunda en términos de la fuerza medida y el desplazamiento medido, como se indica en (9)-(10), respectivamente.

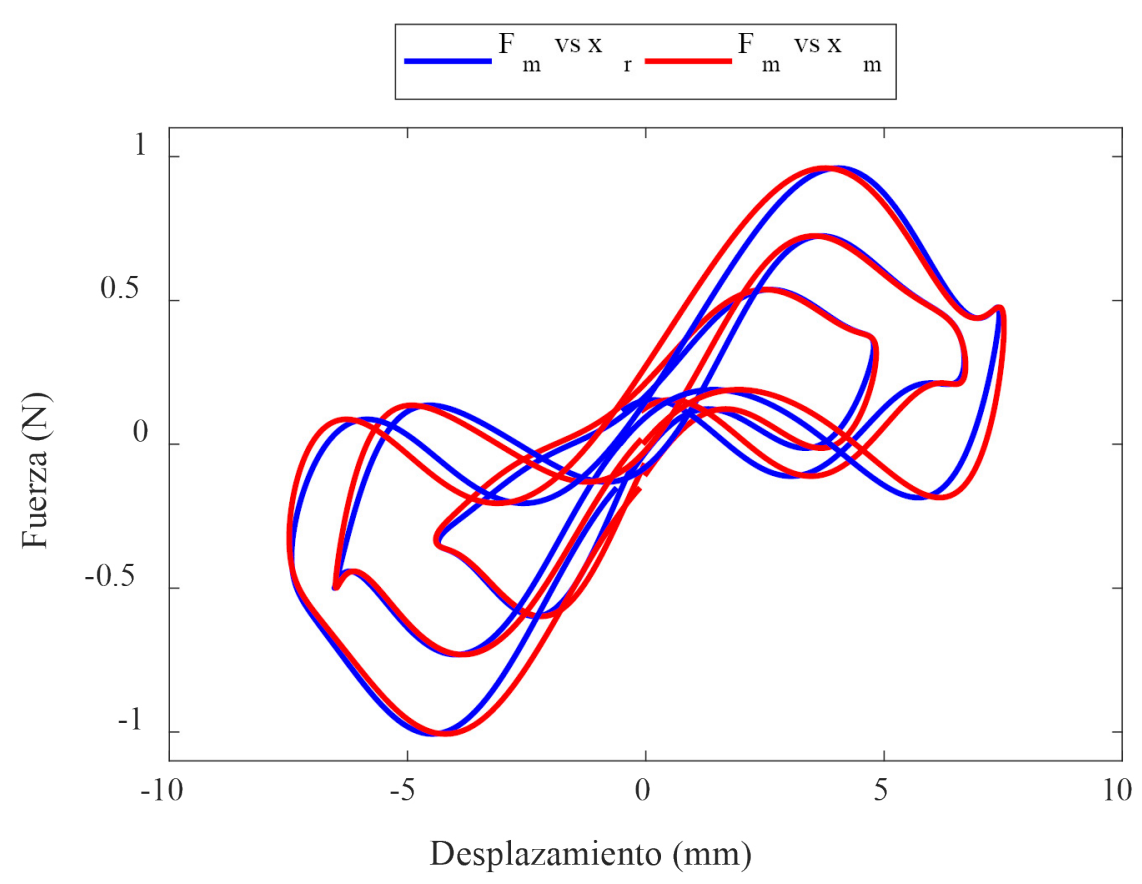

Fig. 11. Ciclos de histéresis. Fuente: Autores.
La Fig. 11 muestra tres ciclos de histéresis del NT$\mathrm{MD}$, para ambas configuraciones de energía, tomados alrededor de la resonancia de la RTHS (Fig. 9). Las diferencias en el área de los ciclos de histéresis calculado y medido se ven reflejadas en el índice de error de energía, como se muestra en la Fig. 12. Este indicador reportó un error final del 8\%. Es decir, que el desplazamiento aplicado a la subestructura experimental fue ligeramente mayor que el calculado, como se observa en algunas zonas de los ciclos de histéresis de la Fig. 11. Este incremento del desplazamiento medido fue ocasionado por el compensador de delay del sistema de transferencia, ya que dicho compensador amplifica el sobrepaso del actuador, mientras aumenta su velocidad de respuesta. Este resultado coincide con lo calculado por la amplitud generalizada, la cual presentó un valor ligeramente mayor que 1 .

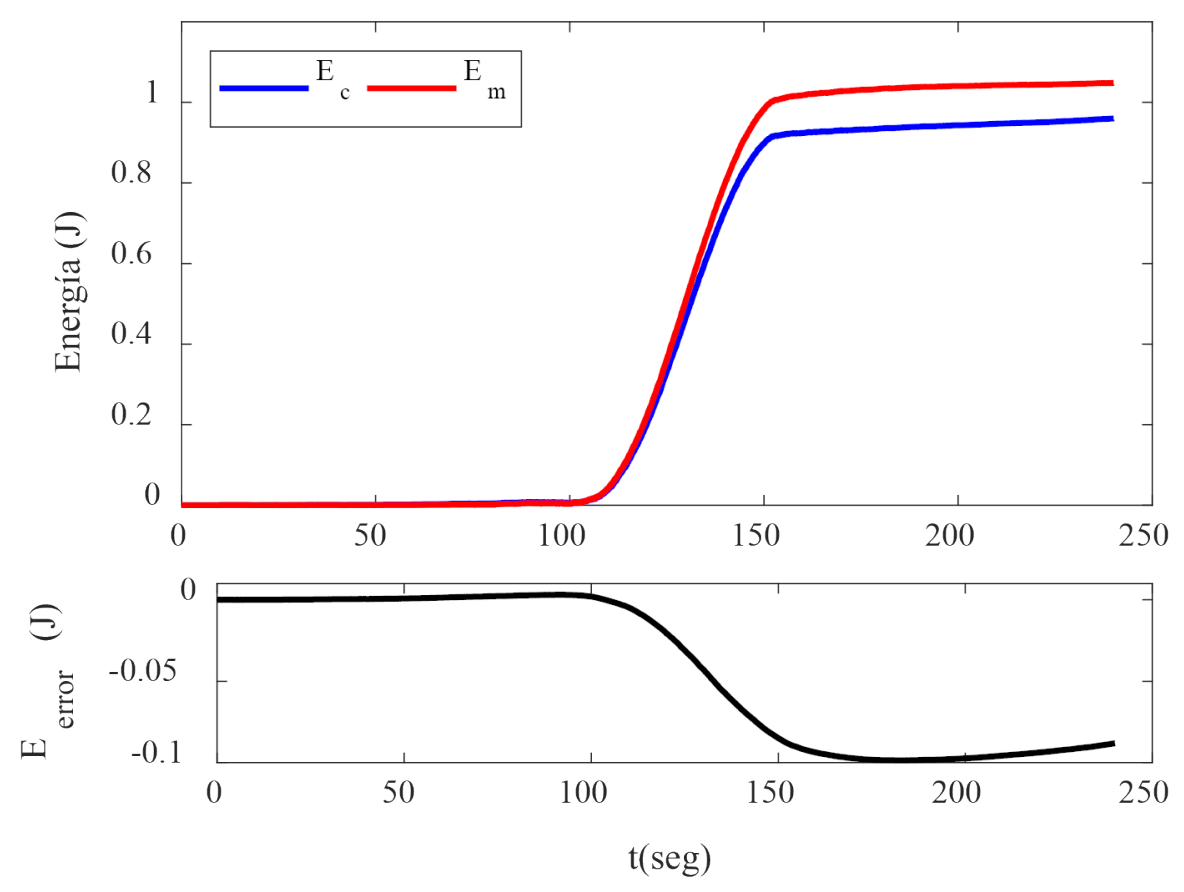

Fig. 12. Indicador de error de energía.

Fuente: Autores.

\section{Comparación entre Mesa Vibratoria}

Al comparar los resultados de la RTHS con los del ensayo de mesa vibratoria se encontró que la aceleración pico de la estructura principal, reportada por la RTHS, fue $20 \%$ mayor que la obtenida con el ensayo de mesa vibratoria. Por otro lado, la aceleración RMS de la RTHS fue un 1\% mayor que la obtenida del ensayo convencional. Sin embargo, al comparar la aceleración del sistema estructura-NTMD, medida en la parte superior de la estructura, se halló un ajuste del $44 \%$ entre ambas técnicas. Este nivel de ajuste fue ocasionado por i) la limitada representación de la subestructura experimental (ajuste del 86\%) y ii) el nivel de ruido registrado en la estimación de la fuerza del NTMD. Sin embargo, la RTHS logró estimar adecuadamente la respuesta RMS del sistema estructura-NTMD y sobreestimó su respuesta pico, en comparación con los resultados obtenidos de los ensayos de mesa vibratoria. 
La Fig. 13 muestra la respuesta en el dominio del tiempo y el de la frecuencia de la estructura con control para las evaluaciones con mesa sísmica y RTHS, donde se puede observar que el NTMD aportó mayor amortiguamiento en el segundo caso (RTHS) que en el primero (mesa vibratoria). Particularmente, la fuerza medida del NTMD durante la RTHS ingresó directamente a la subestructura numérica, con su respectivo nivel de ruido. En consecuencia, el desplazamiento de esta subestructura, afectado por el ruido, fue tomado como el desplazamiento de referencia, lo cual redujo la respuesta del sistema estructura-NTMD, especialmente en la zona de resonancia, y produjo la sobrestimación del amortiguamiento del sistema controlado.

\section{Conclusiones}

Para las dos técnicas descritas en este artículo (mesa vibratoria y RTHS), el sistema estructura-NTMD presentó un comportamiento distinto al de una estructura con un TMD lineal, la cual presenta dos picos en la respuesta en frecuencia. Esto fue ocasionado por el comportamiento no lineal del NTMD.

Los resultados experimentales con mesa vibratoria mostraron al NTMD, con una razón de masas del 10\%, alcanzando reducciones hasta del 77\% y $63 \%$ para las aceleraciones pico y RMS, con respecto a la estructura sin control. Adicionalmente, aumentó más de 6 veces la razón de amortiguamiento de la estructura principal.

Los resultados obtenidos con RTHS mostraron que el NTMD, con una razón de masas del 10\%, alcanzó reducciones de hasta el 73\% y $63 \%$ para las aceleraciones pico y RMS, con respecto a la estructura sin control. Ambas técnicas presentaron una reducción RMS del $63 \%$ y una reducción pico del $75 \%$, en promedio, lo cual demostró una correspondencia admisible entre ellas.
De acuerdo con los indicadores de evaluación del sistema de transferencia se encontró que este presentó un alto nivel de seguimiento. No obstante, las diferencias obtenidas entre la técnica convencional y la RTHS fueron debidos a las limitaciones del modelo de la subestructura numérica, y a los niveles de ruido presentes en la estimación de la fuerza de la subestructura experimental. Sin embargo, la RTHS logró estimar adecuadamente la respuesta RMS del sistema estructura-NTMD y sobreestimó su respuesta pico, en comparación con el ensayo de mesa vibratoria.

Las RTHS tienen la capacidad de estimar adecuadamente el desempeño de sistemas de control en la mitigación de vibraciones. Por lo tanto, esta técnica es una alternativa factible para el fortalecimiento de la investigación en control estructural, aun en países que no cuentan con la infraestructura necesaria para realizar pruebas en mesa vibratoria a escala completa.

\section{Trabajos Futuros}

Los trabajos futuros a este estudio se enfocarán en la combinación de RTHS con actualización de modelos en línea para estudiar el efecto del NTMD en estructura a escala completa. En estos estudios se incluirán variaciones en las propiedades dinámicas de la estructura principal, debidos a daños en los elementos estructurales y no estructurales de la edificación. Adicionalmente, se considerarán como excitaciones diversos eventos sísmicos y cargas eólicas registrados, evaluando diferentes ángulos de aplicación de la excitación. También se desarrollará un NTMD que pueda controlar varios modos de vibración y se realizará un estudio paramétrico de las características del disipador del NTMD utilizando dinámica computacional de fluidos.
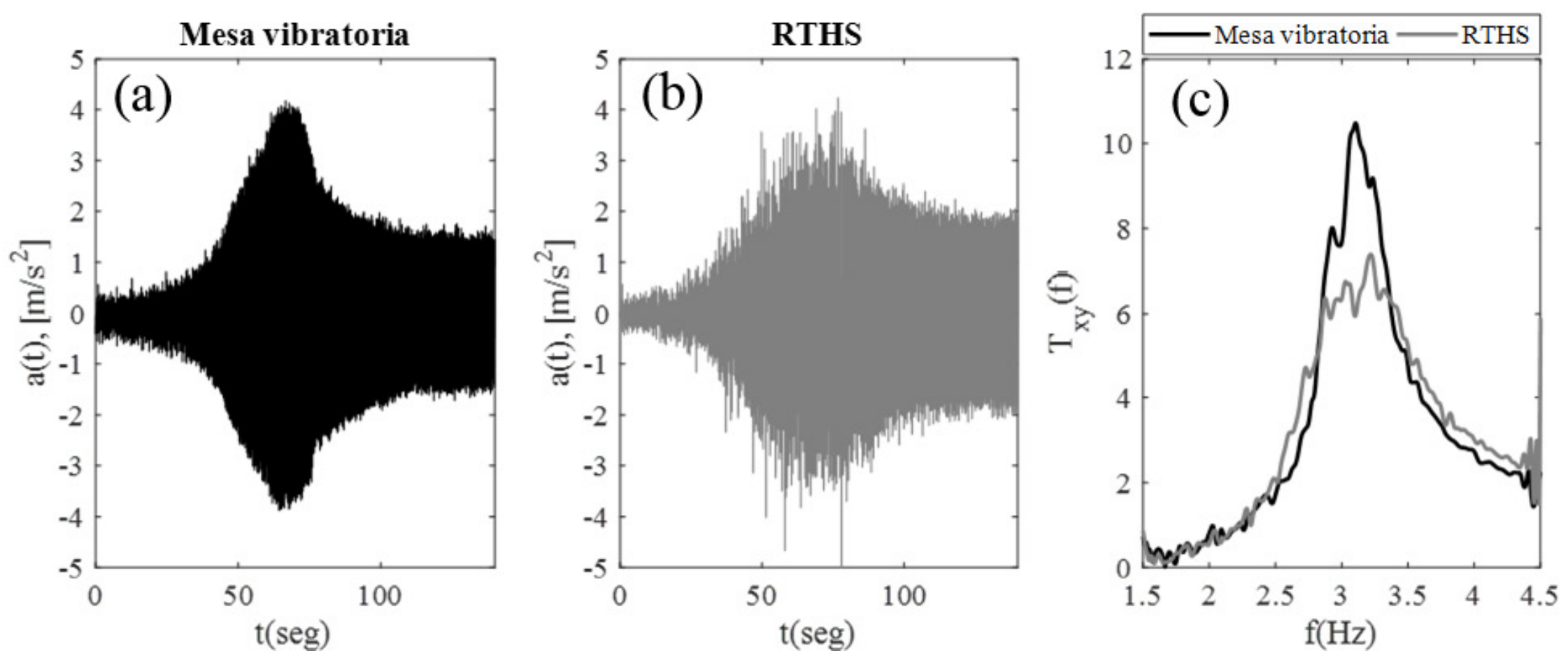

Fig. 13. Comparación de la RTHS y el ensayo de mesa vibratoria.

Fuente: autores. 


\section{FinANCIAMiento}

El financiamiento de esta investigación fue otorgado por parte de la Universidad del Valle (Colombia) a través del proyecto titulado "Evaluación de la respuesta sísmica de estructuras utilizando simulaciones híbridas en tiempo real (Centro de Información: 2965)". Esta investigación contó con el acompañamiento y la asesoría de Purdue University (Estados Unidos). Fecha de inicio: agosto de 2017.

\section{REFERENCIAS}

[1] J. A. Oviedo and M. D. P. Duque, "Status of seismic response control techniques in Colombia," Rev. EIA, vol. 2009, no. 12, pp. 113-124, Jan. 2009.

[2] A. Filiatrault and C. Christopoulos, Principles of passive supplemental damping and seismic isolation. IUSS Press, Pavia, Italy, 2006.

[3] C. Sun, S. Nagarajaiah and A. J. Dick, "Experimental investigation of vibration attenuation using nonlinear tuned mass damper and pendulum tuned mass damper in parallel," Nonlinear Dyn., vol 78, no. 4, pp. 26992715, Aug. 2014. https://doi.org/10.1007/s11071-0141619-3

[4] G. Gatti, "Fundamental insight on the performance of a nonlinear tuned mass damper," Meccanica, vol. 53, no. 1-2, pp. 111-123, Jul. 2018. https://doi.org/10.1007/ s11012-017-0723-0

[5] V. Gattulli and A. Luongo, "Nonlinear tuned mass damper for self-excited oscillations," Wind Struct., vol. 7, no. 4, pp. 251-264, Aug. 2004. https://doi.org/10.12989/ was. 2004.7.4.251

[6] Y. R. Wang, C. K. Feng and S. Y. Chen, "Damping effects of linear and nonlinear tuned mass dampers on nonlinear hinged-hinged beam," J. Sound Vib., vol. 430, pp. 150-173, Sep. 2018. https://doi.org/10.1016/j. jsv.2018.05.033

[7] B. Farshi and A. Assadi, "Development of a chaotic nonlinear tuned mass damper for optimal vibration response," Commun. Nonlinear Sci. Numer. Simul., vol. 16, no. 11, pp. 4514-4523, Nov. 2011. https://doi. org/10.1016/j.cnsns.2011.02.011

[8] K.-C. Chen, J.-H. Wang, B.-S. Huang, C.-C. Liu, and W.-G. Huang, "Vibrations of the TAIPEI 101 skyscraper caused by the 2011 Tohoku earthquake, Japan," Earth, Planets Sp., vol. 64, no. 12, pp. 1277-1286, Jan. 2013.

[9] P. V. B. Guimarães, M. V. G. de Morais and S. M. Avila, "Tuned Mass Damper Inverted Pendulum to Reduce Offshore Wind Turbine Vibrations," in Vibration Engineering and Technology of Machinerym, J. K. Sinha, Ed., Mánchester: University of Manchester, UK., 2015, pp. 379-388. https://doi.org/10.1007/978-3-319-099187_34

[10] G. Mosqueda, B. Stojadinovic and S. A. Mahin, "Energy-based procedure for monitoring experimental errors in hybrid simulations," in $8 N C E E$, 100th Anniversary Earthquake Conference, San Francisco, CA, Apr. 18-22 2006, pp. 1535-1544

[11] G. Mosqueda, B. Stojadinovic and S. A. Mahin, "Realtime error monitoring for hybrid simulation. Part I: methodology and experimental verification," J. Struct. Eng., vol. 133, no. 8, pp. 1100-1108, Aug. 2007. https:// doi.org/10.1061/(ASCE)0733-9445(2007)133:8(1100)
[12] A. Maghareh, A. I. Ozdagli and S. J. Dyke, "Modeling and implementation of distributed real-time hybrid simulation," in NCEE 2014, 10th U.S. National Conference on Earthquake Engineering: Frontiers of Earthquake Engineering, Anchorage, Alaska, Jul. 21-25, 2014. https://doi.org/10.4231/D32B8VC4F

[13] Y. Qian, G. Ou, A. Maghareh and S. J. Dyke, "Parametric identification of a servo-hydraulic actuator for real-time hybrid simulation," Mech. Syst. Signal Process., vol. 48, no. 1-2, pp. 260-273, Oct. 2014. https:// doi.org/10.1016/j.ymssp.2014.03.001

[14] M. L. Brodersen, G. Ou, J. Høgsberg and S. Dyke, "Analysis of hybrid viscous damper by real time hybrid simulations," Eng. Struct., vol. 126, pp. 675-688, Nov. 2016. https://doi.org/10.1016/j.engstruct.2016.08.020

[15] R. Zhang, P. V Lauenstein and B. M. Phillips, "Real-time hybrid simulation of a shear building with a uni-axial shake table," Eng. Struct., vol. 119, pp. 217-229, Jul. 2016. https://doi.org/10.1016/j.engstruct.2016.04.022

[16] J. T. Wang, Y. Gui, F. Zhu, F. Jin and M. X. Zhou, "Real-time hybrid simulation of multi-story structures installed with tuned liquid damper," Struct. Control Heal. Monit., vol. 23, no. 7, pp. 1015-1031, Dec. 2016. https:// doi.org/10.1002/stc. 1822

[17] F. Zhu, J. T. Wang, F. Jin and L. Q. Lu, "Real-time hybrid simulation of full-scale tuned liquid column dampers to control multi-order modal responses of structures," Eng. Struct., vol. 138, pp. 74-90, May. 2017. https://doi. org/10.1016/j.engstruct.2017.02.004

[18] C. Chen, J. M. Ricles, T. L. Karavasilis, Y. Chae and R. Sause, "Evaluation of a real-time hybrid simulation system for performance evaluation of structures with rate dependent devices subjected to seismic loading," Eng. Struct., vol. 35, pp. 71-82, Feb. 2012. https://doi. org/10.1016/j.engstruct.2011.10.006

[19] M. S. Williams and A. Blakeborough, "Laboratory testing of structures under dynamic loads: An introductory review," Philosophical Transactions of the Royal Society A: Mathematical, Physical and Engineering Sciences, vol. 359, no. 1786. pp. 1651-1669, Sep. 2001. https://doi. org/10.1098/rsta.2001.0860

[20] W. J. Chung, C. B. Yun, N. S. Kim and J. W. Seo, "Shaking table and pseudodynamic tests for the evaluation of the seismic performance of base-isolated structures," Eng. Struct., vol. 21, no. 4, pp. 365-379, Apr. 1999. https://doi.org/10.1016/S0141-0296(97)00211-3

[21] G. Ou, S. J. Dyke and A. Prakash, "Real time hybrid simulation with online model updating: An analysis of accuracy," Mech. Syst. Signal Process., vol. 84, Part. B. pp. 223-240, Feb. 2017. https://doi.org/10.1016/j.ymssp.2016.06.015

[22] X. Gao, N. Castaneda and S. J. Dyke, "Real time hybrid simulation: From dynamic system, motion control to experimental error," Earthq. Eng. Struct. Dyn., vol. 42, no. 6, pp. 815-832, Aug. 2013. https://doi.org/10.1002/ eqe. 2246

[23] G. Ou, A. Prakash and S. Dyke, "Modified Runge-Kutta Integration Algorithm for Improved Stability and Accuracy in Real Time Hybrid Simulation," J. Earthq. Eng., vol. 19, no. 7, pp. 1112-1139, Jun. 2015. https://doi.org/1 0.1080/13632469.2015.1027018.

[24] G. Ou, A. I. Ozdagli, S. J. Dyke and B. Wu, "Robust integrated actuator control: Experimental verification and real-time hybrid-simulation implementation," Earthq. Eng. Struct. Dyn., vol. 44, no. 3, pp. 441-460, Oct. 2015. https://doi.org/10.1002/eqe.2479 
[25] A. Friedman et al., "Large-scale real-time hybrid simulation for evaluation of advanced damping system performance," J. Struct. Eng., vol. 141, no. 6, p. 04014150 , Jul. 2015. https://doi.org/10.1061/(ASCE)ST.1943-541X. 0001093

[26] C. Riascos, J. Marulanda and P. Thomson, "Semi-active tuned liquid column damper implementation with realtime hybrid simulations," Active and Passive Smart Structures and Integrated Systems 2016, vol. 9799, p. 979919, Apr. 2016. https://doi.org/10.1117/12.2220004

[27] G. Mosqueda, B. Stojadinović and S. A. Mahin, "Implementation and accuracy of continuous hybrid simulation with geographically distributed substructures," Earthq. Eng. Res. Center, University of California, Berkeley, CA, Tech. Rep. UCB/EERC, 2005.

[28] R. Christenson et al., "Hybrid Simulation: A Discussion of Current Assessment Measures," Earthq. Eng. Res. NSF, NEES, West Lafayette, Indiana, Tech. Rep. Cmmi, 2014.

[29] A. Y. Tuan and G. Q. Shang, "Vibration control in a 101-storey building using a tuned mass damper," J. Appl. Sci. Eng., vol. 17, no. 2, pp. 141-156, Jan. 2014. https:// doi.org/10.6180/jase.2014.17.2.05

[30] R. N. Jabary and G. S. P. Madabhushi, "Tuned Mass Damper Positioning Effects on the Seismic Response of a Soil-MDOF-Structure System," J. Earthq. Eng., vol. 22, no. 2 , pp. 281-302, Jan. 2018. https://doi.org/10.1080/136 32469.2016 .1224743

[31] J. L. Almazán, J. C. De la Llera, J. A. Inaudi, D. LópezGarcía and L. E. Izquierdo, "A bidirectional and homogeneous tuned mass damper: A new device for passive control of vibrations," Eng. Struct., vol. 29, no. 7, pp. 1548-1560, Jul. 2007. https://doi.org/10.1016/j.engstruct.2006.09.005

[32] F. Weber, C. Boston and M. Maślanka, "An adaptive tuned mass damper based on the emulation of positive and negative stiffness with an MR damper," Smart Mater. Struct., vol. 20, no. 1, Dec. 2011. https://doi. org/10.1088/0964-1726/20/1/015012

[33] D. C. Johnson, "Mechanical Vibrations," Nature, vol. 169, no. 641, pp. 271-288, Abr. 1952. https://doi. org/10.1038/169641b0

[34] T. T. Soong and G. F. Dargush, Passive energy dissipation systems in structural engineering, New York, USA MCEER, 1997.
Carlos Andrés Riascos González tiene estudios de pregrado de la Universidad del Valle (Colombia), donde recibió el título de Ingeniero Civil. Actualmente es candidato a Doctor en Ingeniería en dicha universidad. En el marco de sus estudios doctorales realizó una pasantía de investigación en Purdue University (USA) donde fue capacitado para la implementación de simulaciones híbridas en tiempo real. https://orcid. org/0000-0003-4877-9976

Peter Thomson es profesor de la Escuela de Ingeniería Civil y Geomática de la Universidad del Valle (Colombia). Recibió su título de Ingeniero Aeroespacial de University of Minnesota (USA), sus títulos de Maestría en Ingeniería Aeroespacial y Doctorado en Ingeniería Aeroespacial y Mecánica los recibió de la misma University of Minnesota. Es cofundador y actual director del grupo de investigación en Ingenierías Sísmica, Eólica, Geotécnica y Estructural (G-7) de la Universidad del Valle. Adicionalmente, ha dirigido un gran número de proyectos relacionados con estudios dinámicos de estructuras civiles y aeroespaciales. https:// orcid.org/0000-0002-9404-0710

Shirley J. Dyke es profesora de Ingeniería Mecánica y profesora de ingeniería civil en Purdue University (USA). Recibió su B.S. en Ingeniería Aeronáutica y Astronáutica de la University of Illinois (USA), y su Ph.D. en Ingeniería Civil de la Universidad de Notre Dame (USA). Es co-líder de tecnologías de la información para la red financiada por NSF para la simulación de ingeniería sísmica (Network for Earthquake Engineering Simulation-NEES). Sus esfuerzos de investigación han abordado una variedad de temas relacionados con el desarrollo e implementación de estructuras "inteligentes", incluidas tecnologías de control innovadoras para la mitigación de peligros naturales, monitoreo de salud estructural y métodos de simulación híbrida en tiempo real. También es fundadora del Laboratorio de Sistemas de Infraestructura Inteligente en el Laboratorio Bowen de Purdue. https://orcid. org/0000-0003-3697-992X 